\title{
Profile of Dermatophytes in Teaching Hospital and Tertiary Care Hospital from Northern India
}

\author{
Sikander Chirag ${ }^{1}$, Suharshi Gupta ${ }^{2}$ and Shashi Sudan Sharma ${ }^{3}$ \\ Government Medical College, JAMMU, India \\ *Corresponding author
}

\section{A B S T R A C T}

\section{Keywords}

Dermatophytes, Direct microscopy, Tinea cruris, Tinea corporis

Article Info

Accepted:

08 January 2020

Available Online:

10 February 2020
Dermatophytoses refer to superficial fungal infection of keratinophilic dermatophytes. It is common in tropics and in areas with high rates of humidity. The main objective of this study was to assess the clinical profile of dermatophytic infection and to identify the species of fungi and that are prevalent in this region. A total of 77 patients clinically suspected for dermatophytosis were selected for the study. Direct microscopy in $10 \%$ potassium hydroxide $(\mathrm{KOH})$ and culture was done in each case. Out of 77 patients, Tinea cruris (40) was the most common clinical type followed by Tinea corporis (20). Trichophyton rubric was the predominant species isolated (36) in all clinical types followed by Trichophyton mentagrophytes (17).

\section{Introduction}

Dermatophytes are a distinct group of fungi that infect the keratinised tissues like skin, hair and nails of humans and cause different types of cutaneous infections. They are keratinolytic and keratinophilic fungi. Dermatophytic infections are one of the most common skin infections countered by general practitioners and dermatologists. Fungi in this group are known as ringworm fungi.(1)They are classified into three anamorphic genera, Epidermophyton, Microsporum and Trichhophyton.(2). Species of all three groups can cause human infections(3). They can be anthrophilic, zoophilic and geophilic. They are responsible for dermatophytosis which are superficial mycosis affecting skin (Tinea corporis, Tinea cruris, Tinea pedis), hair (Tinea capitis), beard (Tinea barbae).In rare cases they may also cause deep infections. Their severity depends on site of infection, sensitivity of host and cryptogenic potential(4).Dermatophytes uses keratin as nitrogen source and have three genera.They are commonly associated with travelling, immigration, in army camps and migration of humans with poverty, poor hygiene $(5,6)$ 
Identification of dermatophytes upto species level is very important as for treatment and clinical correlation concerns. Rapid identification of dermatophyte species and knowledge of their host preference plays important role in management.The varied clinical face of tinea, which result in diagnostic attenuation and control led to increase work in rapid identification of dermatophytes.(7)

The main aim of this study was to bring in vision the profile of dermatophytes from this region of northern India In the department of microbiology GMC Jammu.

\section{Materials and Methods}

This study of Dermatophytosis was carried out in Department of microbiology govt Medical college Jammu over a period of one year.

Study population included 77 patients clinically suspected of having dermatophytosis randomly selected over a period of one year. A detailed clinical history including age, sex, socioeconomic status, occupation, duration of disease, type of lesion, family history, contact with animals or soil were elicited and recorded in all cases. General physical and systemic examination were conducted and investigations like haemoglobin, total count, differential count, blood sugar and liver function tests were done whenever necessary. Immunocompromised, infants and those over 60 yrs of age were excluded from this study.

\section{Specimen collection}

Skin -Area affected was swabbed with $70 \%$ alcohol allowed to dry and skin scrapings were taken from border areas with scalpel in black paper envelope.
Scalp-Hairs were epilate with a flame sterilised forceps and border area was scraped to collect epidermal scales.

Nail-Nails were cleaned with $70 \%$ alcohol and upper portion of infected nail was scraped away and material was obtained from deeper part of distal end of nail.

All samples were collected on sterile black paper envelope.

The samples of skin and hair were subjected to $10 \% \mathrm{KOH}$ and $20 \% \mathrm{KOH}$ preparation for nail.After 15-20 minutes the specimen was examined for presence of hyphae or arthrospores.

\section{Culture}

For primary isolation sabourauds dextrose agar containing cycoheximide and chloramphenicol was used irrespective of demonstration of fungal elements on $\mathrm{KOH}$ mount was used.One tube with antibiotic and other without antibiotic were incubated at 27 c.dermatophyte etst media was used as selective media.

LCB mount was made on all culture growths .Slide culture was done to study the micromorphology of microconidia, macroconidia, nature of sporulation, special structures such as spirals, pectinate, racquet hyphae and chlamydospores. Special tests were performed when necessary like hair perforation test and biochemical tests like urease test for species identification.

\section{Results and Discussion}

A total of 77 patients were enrolled in study. Out of these 47 were males and 30 were females. The present study revealed that dermatophytosis was more common in males $(61 \%)$ than females $(39 \%)$. $\mathrm{KOH}$ mount was 
positive for fungal elements in 68 cases and negative in 9 cases. Culture was positive for fungal isolates in 57 cases and negative in 20 cases. Isolates which showed culture positivity were also positive for hyphae on $\mathrm{KOH}$ mount.(57), Only 11 isolates which were $\mathrm{KOH}$ positive didn't showed any growth. Tinea cruris was most (40 cases) clinical pattern observed followed by Tinea corporis (20 cases), Tinea ungium(8 cases), Tinea manuum (5 cases), Tinea capitis ( 2 cases) and Tinea pedis(2).

Out of 57 isolates grown on culture in our study T.rubrum was most common(36), followed by T.mentagrophyte (17) followed by one each for Epidermophyton flocossum, Microsporum gypseum, T.tonsurans and T.Verrucosum.

In the present study, all the three genera of dermatophytes i.e, Trichophyton, Epidermophyton and Microsporum were isolated as causative agent.

In present study 77 clinically diagnosed cases of Dermatophytosis were studied out of which 69 were skin scrapings, 6 were nail clippings and 2 were hair stubs.

Various clinical types of dermatophytosis-
1)Tinea cruris-40
2)Tinea corporis-20
3)Tinea ungium-8
4)Tinea manuum-5
5)Tinea capitis-2
6)Tinea pedis-2

Out of 77 clinical samples 57 samples which were $\mathrm{kOH}$ positive also showed growth on fungal media.

Table.1 Clinical types and causative dermatophytes

\begin{tabular}{|l|c|c|c|c|c|c|}
\hline Dermatophytosis & T.r & T.M & T.T & T.Ve & E.F & M.G \\
\hline T.cruris & 21 & 9 & 1 & 1 & 1 & 0 \\
\hline T.capitis & 0 & 0 & 0 & 0 & 0 & 1 \\
\hline T.ungium & 2 & 4 & 0 & 0 & 0 & 0 \\
\hline T.pedis & 5 & 1 & 0 & 0 & 0 & 0 \\
\hline T.manuum & 1 & 0 & 0 & 0 & 0 & 0 \\
\hline T.corporis & 7 & 3 & 0 & 0 & 0 & 0 \\
\hline
\end{tabular}

T.r=Trichophyton rubric, T.M=Trichophyton mentagrophytes, T.T=Trichophyton tonsurans, T.Ve= Trichophyton verrucosum, E.F= E.floccosum, M.G= M.gypseum

Superficial fungal infections are a world wide problem constituting a large number of cases. Dermatophyte infections are more prevalent in developing world. Hot and humid climate of tropical and subtropical regions are best suited for dermatophyte infections. This study was conducted in GMC Jammu a tertiary care teaching hospital in northern India which shows hot and warm weather favourable for dermatophytic infection. In addition to hot climate in Jammu other factors like tourism, migration of labours and unhygienic practices contributes to this infection. Out of 77 samples received $47(61 \%)$ were males and $30(39 \%)$ were females. The higher incidence in males can be due to greater physical activity and increased sweating. High prevalence in males have also been reported from other studies in India $(8,9)$. Of the total of 77 samples $\mathrm{KOH}$ mount was positive in 68 samples and culture positivity was seen in 57 samples. Similar type of high percentage 
culture positive findings were observed in other studies.(10,11).

In the present study most common type of dermatophytosis studied was Tinea cruris (40 cases), some other studies showed the the similar results.(12).Studies published also showed that Tinea corporis being the most common dermatophytosis((13).In the present study T.rubrum was the commonest dermatophyte isolated from 36 clinical samples. Second most common dermatophyte isolated was T.mentagrophyte from 17 clinical samples.T.rubrum as the commonest isolate was also observed in various other studies(14). T.mentagrophytes isolates were found second in frequency similar to study in south India(13). E.floccosum was the most common etiological agent of dermatophytosis in a study by Pashkir at Tehran(15).In another study by Grover et al in India T.tonsurans was the most common dermatophyte followed by T.rubrum. Overall in our study $90 \%$ of isolates were Trichophyton genera dominated followed by Microsporum and Epidermophyton in agreement with other studies(16).It is important for family physicians to emphasise that clinical diagnosis of dermatophytosis can be unreliable because these infections can have many mimics. Luckily in our study none of contaminant was observed or any other than dermatophytes. $\mathrm{KOH}$ mount positivity was higher than culture so $\mathrm{KOH}$ mount can be used as point of care test before prescribing medications.

In our study T.rubrum was commonest followed by T.mentagrophytes.

In conclusion, present study showed T.cruris as the most common pattern of dermatophytosis followed by T.corporis and T.rubrum as most common agent of dermatophytes in this region of Jammu and Kashmir. This data could help in diagnosis of the disease and spread of disease can be controlled with specific control measures.

\section{References}

Ajello L.A toxonomic review of dermatophytes and related species.Sabouradia 1968;6:147-59

Ajello L.Present day concepts in dermatophytes. Mycopathol 1962; 17:315-24

Bhatia VK,Sharma PC.Epidemiological studies on dermatophytosis in Human patients in Himachal Pradesh, India.Springer Plus 2014

Bindu V,Pavithran K.Clinico-mycological study of dermatophytosis in calicut.Indian $\mathrm{J}$ Dermatol venerol Leprol 2002;68(5):259-61

Bitew A. Dermatophytosis: Prevalance of dermatophytes and non dermatophyte fungi fro patients advanced medical laboratory, Addis ababa, Ethiopia. Dermatol Res Pract 2018

Karmakar SG,Kalla KR.Dermatophytosis in a desert district of Western Rajasthan.Indian J Dermatol Venerol Leprol 1995;61(5):280-83

Mahajan S,Tilak R. Clinicomycological study of dermatophytic infections and their sensitivity to antifungal agents in a tertiary care center. Indian $\mathbf{J}$ Dermatol,Venerol Leprol 2017;83:43640

Nita P, Rashmika D.Dermatomycosis in and around Aurangabad.Indian J Pathol Microbial 1999;42:455-62

Pakshir K,Hashemi J.Dermatophytosis in Karaj,Iran.Indian

Dermatol.2006;51:262-4

Rabell G, Taplin D.Dermatophytes: Their recognition and identification. University of Miami Press;1974

Richardson M.Fungal infection:Diagnosis and management. Oxford, UK:Wiley;2012.p.4 
Sepahvand A,Fallahi S.Dermatophytosis in western part of Iran.Asian journal Biol Sci 2009;2:58-65

Sharma M,Sharma R.Profile of dermatophytic and other fungal infections in Jaipur.Indian J Microbiol 2012

Singh S,Beena PM.Profile of dermatophyte infection in baroda.Indian J Dermatol Venerol Leprol.2003;69:281-3
Sumana MN,Rajagopal V.A study of dermatophytes and their in vitro antifungal sensitivity Indian $\mathrm{J}$ Pathol Microbiol.2002 Apr;45(2):169-72

Topley, WT and Wilson's microbiology and microbial infections. $9^{\text {th }}$ ed london: Hodder Arnold;2005,215-235

\section{How to cite this article:}

Sikander Chirag, Suharshi Gupta and Shashi Sudan Sharma. 2020. Profile of Dermatophytes in Teaching Hospital and Tertiary Care Hospital from Northern India. Int.J.Curr.Microbiol.App.Sci. 9(02): 1116-1120. doi: https://doi.org/10.20546/ijcmas.2020.902.130 